\title{
An automated image dehazing method for flood detection to improve flood alert monitoring system
}

\author{
Chien-Hao Tseng ${ }^{1}$, Lun-Chi Chen ${ }^{1}$, Jyh-Horng Wu ${ }^{1}$, Fang-Pang Lin ${ }^{1}$ and Ruey-Kai Sheu ${ }^{2 *}$ \\ ${ }^{I}$ National Center for High-Performance Computing, National Applied Research Laboratories, Taiwan (ROC). \\ ${ }^{2}$ Department of Computer Science, Tung Hai University, Taichung, Taiwan (ROC).
}

Revised: 18 January 2018; Accepted: 23 March 2018

\begin{abstract}
Flood hazard prevention and mitigation is an emergent environmental problem. Traditional flood monitoring devices operated under adverse environmental conditions are typically influenced by changes in weather conditions such as haze, fog and rain. Consequently, the video images obtained from such devices are often blurred or damaged, increasing the possibility of erroneous assessments in hazard mitigation processes. To ensure the efficient use of image analysis technology to improve degraded images captured under hazy weather conditions, this study proposes an automated single image dehazing method for flood monitoring. This method is based on the dark channel prior for the removal of haze from a single image. The concept of the dark channel prior is that most local patches in haze-free outdoor images contain some pixels having extremely low intensities in at least one colour channel. When this dark channel prior is used, the thickness of haze in the image can be directly estimated and a high-quality haze-free image can be obtained. The proposed method can be used to accurately improve flood detection and monitoring results. The ability to detect and remove haze from a single image is a crucial function when applying automated computer vision to disaster-monitoring applications. The experimental results show that the proposed method can efficiently alleviate the degradation of surveillance images and effectively identify flooded regions in particular areas.
\end{abstract}

Keywords: Flood monitoring, hazard mitigation, haze imaging model, image dehazing.

\section{INTRODUCTION}

Flooding is a major disaster occurring in various parts of the world. Heavy rain, hurricanes, and tsunamis are the main causes of flooding, and heavy rain is the most dominant among these factors. Prolonged rainfall causes rivers to overflow, consequently flooding the surrounding areas. Although video surveillance systems can be used to forecast rainfall or track storm paths precisely, the availability of real-time monitoring data such as water flow, flood level, and water level is essential in making reasonable decisions on necessary flood prevention measures.

Recently, video surveillance systems have been effectively used for monitoring flood events. Flood monitoring at embankments, bridges, levees and dams is essential for flood prevention. Various methods can be used to monitor and prevent flood events. One of the most challenging problems in flood response is the precise localisation of flood risk areas, which is typically conducted using early warning systems (EWSs) for flood prevention and disaster management. EWSs have been used for monitoring flooding by employing remote sensing technologies such as satellite imaging and electronic sensors installed near rivers and seaports (Basha et al., 2008; Krzhizhanovskaya et al., 2011). Flood monitoring using real-time sensors is a non-structural flood control measure. Losses caused by flooding can be mitigated or prevented by using measures such as flood monitoring, forecasting, simulation, evaluation and analysis (Chen, 1990). One non-structural measure, the integration of wireless sensors with web-based decision support systems, has been influential in monitoring, controlling, and assessing natural disasters, particularly flood disasters (Zhang et al., 2002; Sapphaisal, 2007).

\footnotetext{
* Corresponding author (rickysheu@thu.edu.tw; (iD https://orcid.org/0000-0002-3014-8095)
} 
Effectively implementing a flood monitoring and warning system is imperative because such a system requires the availability of reliable and related information. Flooding has recently been studied using various considerations and methodologies such as image processing technology (Rani et al., 2011; Selvi \& Sathya, 2014; Wu et al., 2015), wireless sensor networks (Chang \& Guo, 2006; Hughes et al., 2006), and middleware embedded systems (Shukla \& Pandey, 2014). A decision-theoretic methodology for modelling and evaluating forecast response systems can be combined with loss functions and prior knowledge about actual river water levels (Krzysztofowicz \& Davis, 1983). Albanese et al. (2008) have reported that theories derived from mathematical-morphology-based image processing methods can be used to develop automated detection systems that can provide flood warnings. These studies provide great insight into the development of flood forecasting and modelling systems by using remote sensing data and digital image processing techniques. Flood alarm systems are one of the most widely used infrastructures for creating flood monitoring and warning systems. Closed-circuit television (also called video surveillance) systems are another example of such infrastructures.

Flood monitoring generally involves using on-site water-level measurement facilities, such as rainfall observation stations, water level observation stations, and meteorological stations. On-site stations can directly measure the water or rainfall level and provide instant notifications. However, using sensors to directly measure the water level is particularly restricted by the sensor installation location and necessity of frequent maintenance. Furthermore, this direct measurement approach has the disadvantage of returning only water-level information and not visual evidence for assessment. Therefore, the integration of image analysis techniques with flood alarm systems is vital for flood disaster prevention. Although real-time videos of river surroundings or urban areas can be easily obtained from existing video surveillance systems, various uncertainties in the outdoor environment may affect the video quality. Therefore, video surveillance systems are influenced by haze, fog, rain and other atmospheric conditions, which increase the possibility of erroneous assessment.

The removal of weather effects such as haze (Gibson et al., 2012; Guo et al., 2014; Zhai \& Ji, 2015; Patel \& Nakrani, 2016), fog (Chen et al., 2013; Tan et al., 2014), rain (Barnum et al., 2010; Kang et al., 2012), and air pollution (Abeyratne \& Ileperuma, 2006; Attanayaka \&
Wijeyaratne, 2013) from videos or sensors has recently received considerable attention. In the current study, we focused on haze detection and removal from surveillance images. Surveillance images captured from optical devices are usually degraded by turbid media such as haze, smoke and fog. Haze is the most common problem in outdoor scenes because of atmospheric conditions. When the depth of haze in a hazy image is unknown, dehazing the image becomes a challenge. Furthermore, if the available input is only a single hazy image, the problem is under-constrained. Hence, most traditional dehazing approaches (Narasimhan \& Nayar, 2003; Namer et al., 2009) entail using multiple hazy images as inputs or additional prior knowledge. Moreover, polarisationbased methods have been proposed for removing haze effects from two or more images taken under different polarisation conditions (Schechner et al., 2003; Shwartz et al., 2006). Nevertheless, taking multiple images of the same scene is usually impractical in real-time monitoring system applications. He et al. (2010) proposed an effective image prior, called the dark channel prior, for removing haze from a single image. The key concept of the proposed prior is that most local patches in outdoor haze-free images contain certain pixels having extremely low intensity values in at least one colour channel.

In this paper, inspired by a single-image-based dehazing framework based on the dark channel prior methodology, an image -based flood alarm module (IFAM) that can be integrated into an automated single image dehazing algorithm for flood monitoring is proposed. The IFAM is the foundation of the image analysis technique employed in this study to detect and identify flood conditions in real-time video images. In foggy weather during typhoon, the fuzzy images caused by fog or haze have a negative influence on the recognition and detection of water stage at the backend, which render the warning process less effective. Thus, this paper proposes an image dehazing technique to improve the image quality, in order to elevate the accuracy and liability of image analysis on water stage detection. The module is connected to outdoor surveillance cameras installed around high-risk rivers and other places. Moreover, an automatic single image dehazing method was employed based on the dark channel prior to improve the flood detection and monitoring results. The dark channel prior is based on the statistics of outdoor hazefree images. The experimental results indicated that the proposed method can be used to detect and remove haze from a single image and effectively identify flooding in particular areas. 


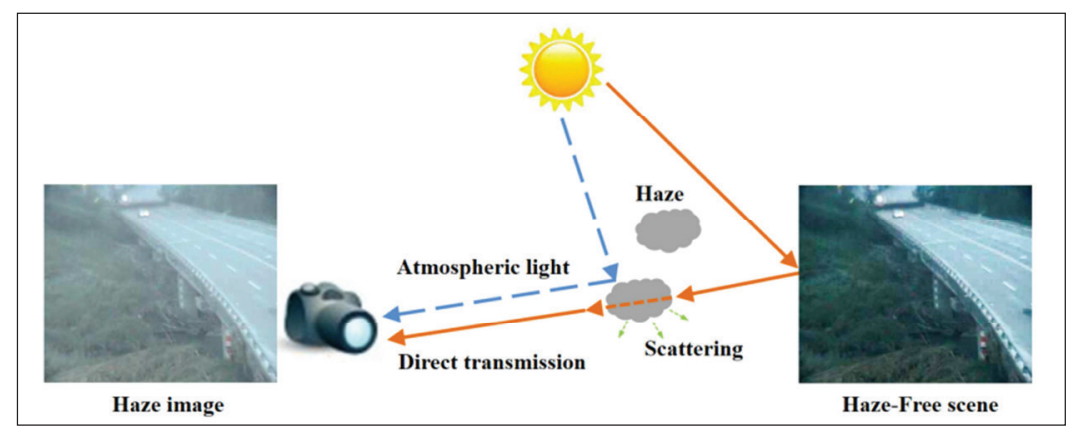

Figure 1: Schematic of the haze imaging model applied to a natural scenario with haze

\section{METHODOLOGY}

\section{Image degradation model and haziness analysis}

In 1976, McCartney proposed a haze imaging model (also called image degradation model) comprising a direct attenuation model and an air light model. The direct attenuation model describes the scene radiance and its decay in the medium, whereas the air light model is composed of previously scattered light and it describes the shift in the scene colours. As illustrated in Figure 1, haze is a turbid medium (e.g., dust particles, tiny water droplets, and molecules) in the atmosphere and degrades outdoor images because of atmospheric absorption and air light scattering. The haze imaging model can be expressed as follows:

$$
\mathrm{I}(x)=\mathrm{J}(x) t(x)+\mathrm{A}(1-t(x))
$$

where $I$ is the observed intensity and the input hazy image, $\mathrm{J}$ is the scene radiance and the restored hazefree image, $\mathrm{A}$ is the global atmospheric light that can be obtained using the dark channel prior, and $t$ is the medium transmission describing the portion of the light that reaches the camera. The major goal of single image dehazing is to recover a haze-free image $\mathrm{J}$, A and $t$ from the input image I. In this model, the term $\mathrm{J}(x) t(x)$ denotes direct attenuation, and the term $\mathrm{A}(1-t(x))$ is called air light. Direct attenuation denotes the scene radiance and its decay in the medium, whereas the air light results from previously scattered light and leads to a shift in the scene colours. This haze image model has been employed in numerous studies involving single image dehazing (Fattal, 2008; Zhang et al., 2010; Xiao \& Gan, 2012).

The dark channel prior is a type of statistic used in processing haze-free outdoor images. It is based on the observation that most local patches in haze-free images contain pixels having extremely low intensities in at least one colour channel. Consider an arbitrary image $\mathrm{J}$; its dark channel $J^{\text {dark }}$ can be expressed as follows:

$$
J^{\text {dark }}(x)=\min _{y \in \Omega(x)}\left[\min _{c \in\{r, g, b\}} J^{c}(y)\right]
$$

where $J^{c}$ is a colour channel of $\mathrm{J}, \Omega(x)$ is a local patch centred at $x, y$ is the index of a pixel in $\Omega(x)$, and $c$ is one of the three colour channels in the RGB (red, green and blue) space.

When the dark channel prior is combined with the haze imaging model, the value of air light $\mathrm{A}$ can be directly estimated to recover a high-quality haze-free image. According to the concept of a dark channel, if $\mathrm{J}$ is an outdoor haze-free image, except for the sky region, the intensity of J's dark channel is low and tends to approach zero (i.e, $\quad J^{\text {dark }} \rightarrow 0$ ), which is called the dark channel prior. In this study, the haze imaging model and dark channel prior were used to remove the effect of haze from video images. The low intensity in the dark channel is mainly attributed to three properties: (1) shadows, (2) colourful objects or surfaces and (3) dark objects or surfaces.

\section{Single image dehazing based on dark channel prior}

He et al. (2010) proposed a single image dehazing method based on the dark channel prior. This method is based on the assumption that the dark channel of a hazy image can approximate the haze density; hence, this study also used the dark channel to estimate the regions with the highest haze densities in the surveillance images. The top $0.1 \%$ brightest pixels in dark channel locations of a hazy image were used, which are usually most haze-opaque. Among these pixels, pixels with the highest intensity values in the hazy image are considered as atmospheric light. 
Once the atmospheric light is known, in a noise-free image, the transmission map can be derived as follows:

$$
t(x)=1-w \min _{y \in \Omega(x)}\left(\min _{c} \frac{I_{c}(y)}{A^{c}}\right)
$$

where $w(0<w \leq 1)$ is a scaling parameter used to maintain an extremely small amount of haze for a distant object, and $c$ represents a specific colour channel (i.e., the red, green or blue channel of the corresponding parameter). Thus, an estimate for the transmission map can be obtained by simply subtracting the dark channel of the normalised image from equation (3).

After the initial haze estimate is obtained, a refinement process is required to suppress halo artifacts. Therefore, $t(x)$ is refined using the matting Laplacian matrix (Levin et al., 2008). This refinement process provides visually satisfactory results, and it was thus used in this study. Finally, the dehazed image is recovered by a simple inversion of equation (1) and solving for J. The haze-free image $\mathrm{J}$ can be recovered as follows:

$\mathrm{J}(x)=\frac{I(x)-A}{\max \left(t(x), t_{0}\right)}+A$

where $t_{0}$ denotes a lower bound of $t(x)$ used to preserve a small amount of haze in extremely dense haze regions. A typical value of $t_{0}$ is 0.1 . Because the scene radiance is usually not as bright as atmospheric light, the surveillance images appear dim after haze removal. Therefore, to identify flooded areas, the exposure of $\mathrm{J}(x)$ was increased.

\section{The proposed image-based flood alarm framework}

The IFAM is a flood alert system that facilitates proactive monitoring, assessment, and early response to rising water levels and associated inundations in near real-time. The IFAM receives video streams from digital camera sensors installed around rivers. The video streams are decomposed into JPEG images and filtered for image enhancement. The module then calculates water levels and assesses the corresponding flood risks. Figure 2 illustrates the proposed flood alert monitoring system. The IFAM combines on-site real-time video images with a backend image processing module to conduct near real-time river overflow and ground inundation analysis. After the water overflow range is calculated by using an image processing module, the system automatically provides flood alarms if the overflow range exceeds the preset warning ranges.

The proposed IFAM-based flood alert monitoring system involves two major steps: image dehazing and detection of flooded areas. The image dehazing process is based on the dark channel prior. This prior can be used to directly estimate the thickness of the haze, and a high-quality haze-free image can be obtained after this estimation. In the detection of flooded areas, image analysis technology is used to evaluate the water level in relation to the preset overflow range. The module then determines whether to send a warning message depending on the calculated results. Details on the operating mechanisms of these modules are provided in the next section.

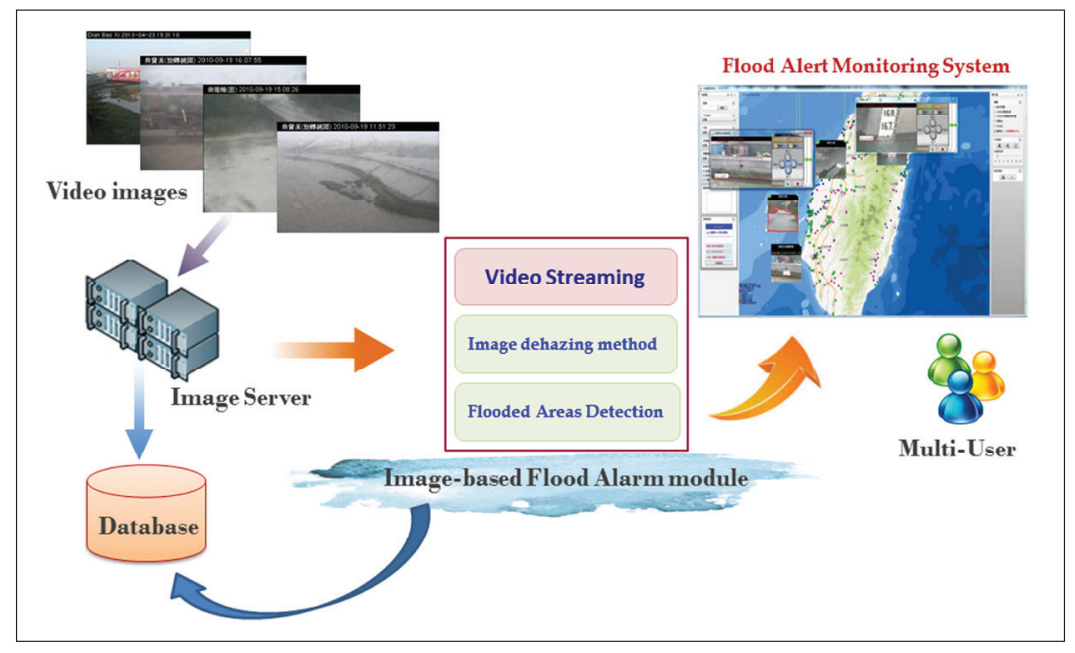

Figure 2: Sketch of the proposed flood-alert monitoring system 


\section{Identifying flooded areas by using image processing module}

In general, in a hazy image, the haze density is higher in deeper regions and lower in shallower regions. To estimate the haze density and remove the haze, the initial haze transmission was first estimated by using the dark channel prior. Furthermore, the atmospheric light values are automatically estimated, and the sky regions are accurately segmented from the image according to their distinctive features. Therefore, the dark channel prior is effective in recovering saturated colours and extracting low-contrast objects. The resulting transmission maps are reasonable. Next, a bilateral filter was applied to obtain a refined haze transmission and to smoothen its small-scale textures. Finally, the haze-free image can be obtained by multiplying the albedo by the atmospheric light according to the haze imaging model. Fattal (2008) proposed an albedo estimation-based method for single image dehazing. The method is able to utilise image prior through a technique called dark channel prior. Figure 3 depicts a flowchart of the described process.

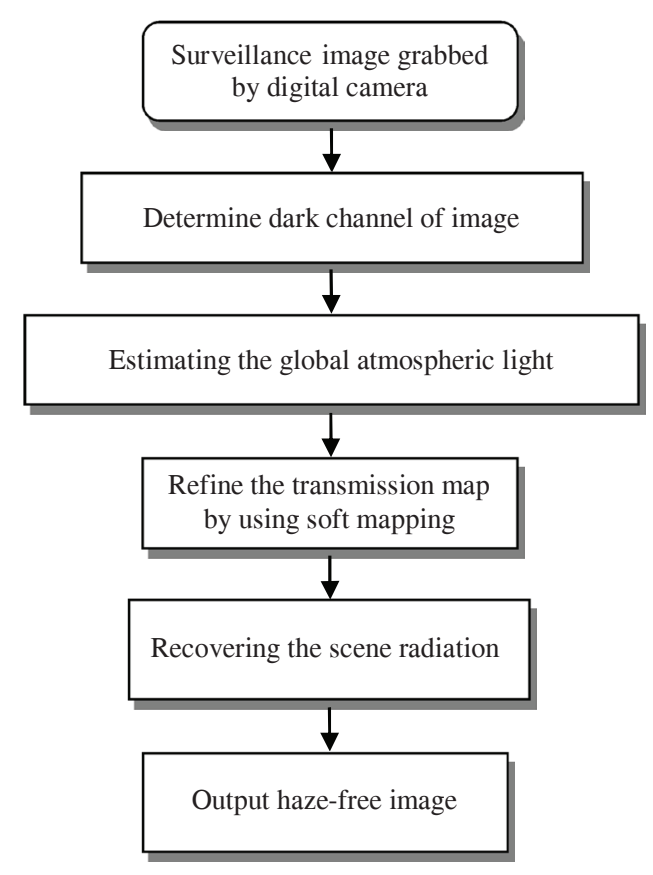

Figure 3: Flowchart of the haze removal method for surveillance images

According to the properties of the resulting haze-free image, it was determined that region-based image classification methods are more suitable for flood region detection compared with pure pixel-based methods.
Therefore, in this study, region-based image identification methods were developed by combining the mean-shift (MS) and region growing (RegGro) algorithms.

The MS algorithm is a powerful nonparametric clustering algorithm involving nonparametric estimation of probability density functions, and it can be used to estimate the local density gradients of similar pixels. The gradient estimates are used in an iterative procedure to determine the peaks in the local density. In the iterative stage of the MS procedure, each pixel is assigned an MS point $M\left(x_{i}\right)$, which is initialised to coincide with the pixel. A multivariate kernel density estimator with a bandwidth matrix $\mathrm{H}$ computed at point $x$ is derived as follows:

$\hat{f}(x)=\frac{1}{n} \sum_{i=1}^{n} K_{H}\left(x-x_{i}\right)$

where the $n=$ data points and $x_{i}$ represent a sample with an unknown density $f$.

$K_{H}(x)=|\mathrm{H}|^{-1 / 2} K\left(\mathrm{H}^{-1 / 2} x\right)$

where $K(x)$ is a $d$-dimensional kernel function satisfying the conditions for asymptotic unbiasedness, consistency, and uniform consistency of the gradient of the density estimate. The term $\mathrm{H}$ is a symmetric positive definite $d \times d$ bandwidth matrix.

If a Gaussian kernel is used, the MS vector becomes

$$
m_{h . K}(x)=\frac{\sum_{i=1}^{n} x_{i} \exp \left(\left\|\frac{x-x_{i}}{h}\right\|^{2}\right)}{\sum_{i=1}^{n} \exp \left(\left\|\frac{x-x_{i}}{h}\right\|^{2}\right)}
$$

The term $m_{h . K}(x)$ is proportional to the normalised density gradient and is always oriented towards the maximal density increase.

The MS algorithm involvs the following steps:

Step 1: Compute the MS vector for point $m_{h, K}\left(x_{i}^{k}\right)$ according to equation (7).

Step 2: Update the current position:

$$
x_{i}^{k+1}=x_{i}^{k}+m_{h, K}\left(x_{i}^{k}\right) .
$$

Step 3: Repeat steps 1 and 2 until convergence; specifically, repeat these steps until the gradient of the density functions at the point is zero (i.e., $\nabla \hat{f}_{h, K}(x)=0$ ). 
The RegGro algorithm is a simple region-based image segmentation algorithm. This algorithm groups pixels into meaningful regions, starting from a specific seed pixel and spreading to neighbours that satisfy the growing rule. The fundamental disadvantage of intensity-based region segmentation is that intensity alone provides no spatial information and the threshold criterion is a single value or a set of grey levels. Hence, in the RegGro algorithm, a compact dynamic mean intensity is used with a settled threshold (intensity distance) to implement the growing rule.

\section{RESULTS AND DISCUSSION}

In this study, the proposed IFAM-based flood alert monitoring system was employed to identify flooded areas. All surveillance images of the flooded areas captured from streaming videos were evaluated. Although the examples that this paper demonstrates can be conducted by human eye, the large dataset will cause tiredness to further influence the accuracy. Therefore, this study can achieve the effectiveness of automated warning by automatically detecting the water stage.
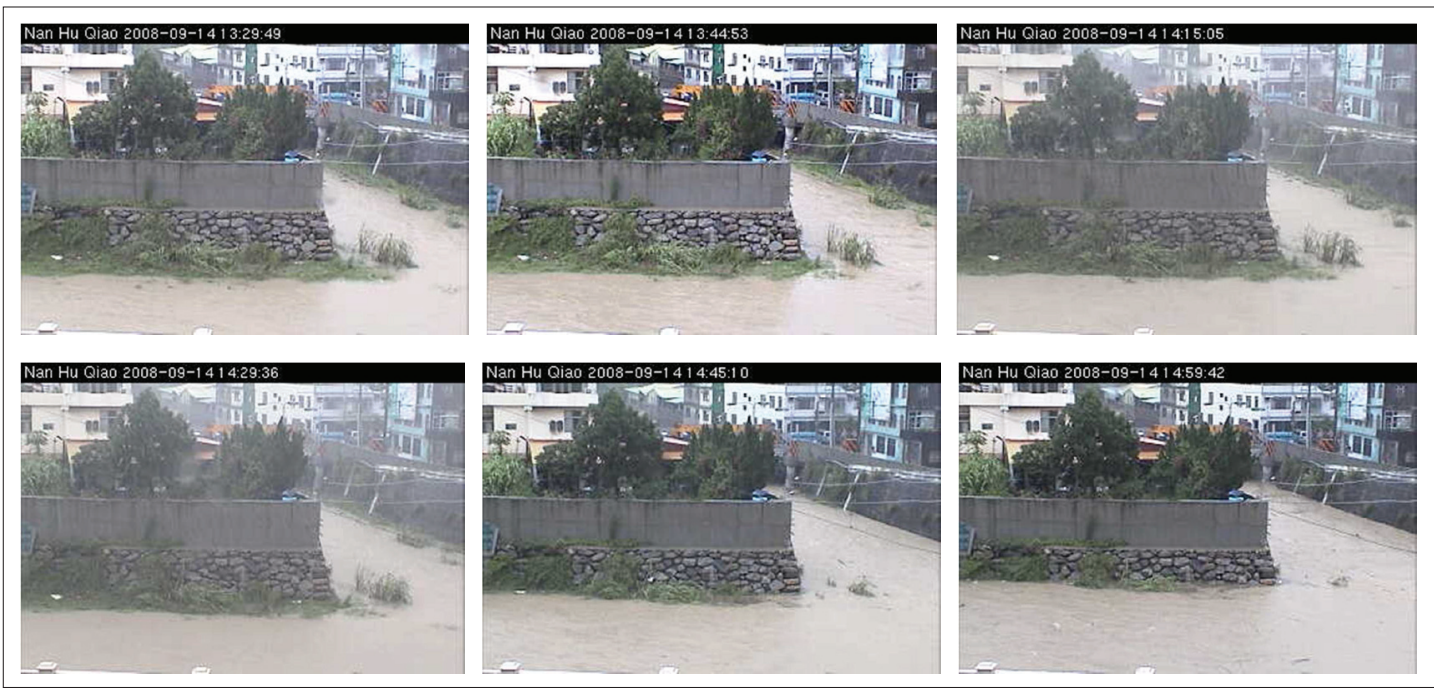

Figure 4: Surveillance image sequence (from top to bottom, left to right) for the Nan-Hu No. 2 bridge dataset
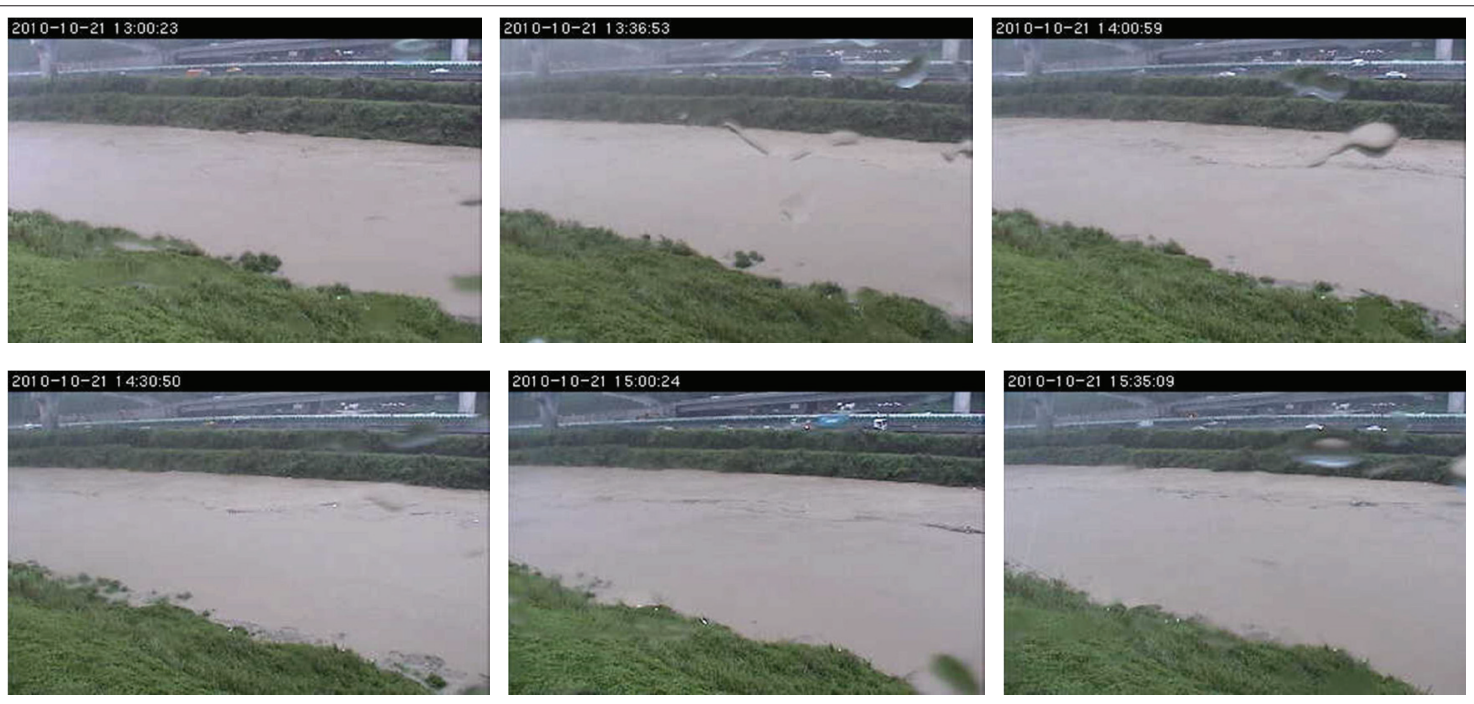

Figure 5: Surveillance image sequences (from top to bottom, left to right) for the Ma-Ling-Keng River dataset 
Furthermore, utilising this image-dehasing technique improves the quality of images, and can further assist the backend module to improve the effectiveness of recognition. All images were in JPEG format, exhibiting a 24-bit colour depth and a spatial resolution of $352 \times 240$. Field experiments were conducted using data from the Nan-Hu No. 2 Bridge and Ma-Ling-Keng River in Taiwan. The Nan-Hu No. 2 Bridge data were recorded during the Typhoon Sinlaku, which induced a rainstorm on September 14, 2008. The video images were captured between noon (when the rain began) and nightfall (when the flood tide occurred). Figures 4 and 5 show the surveillance images of the Nan-Hu No. 2 Bridge and Ma-Ling-Keng River used in the experiments, respectively.

In this study, the proposed IFAM was used to evaluate the test areas in various image sets to determine the optimal image dehazing and flood identification methods. The automated identification method for conducting flood monitoring is based on real-time video images. The performance of the proposed single image dehazing method was evaluated by applying it to recover several hazy images. Figure 6 illustrates the experimental results of the proposed dehazing method, indicating that the proposed method can effectively remove haze, even in a region with high haze density. Therefore, the dark channel prior can be used to directly estimate the thickness of the haze and consequently obtain a highquality haze-free image.
The dehazed images were analysed using the mentioned image analysis techniques to evaluate the water level of the current overflow range. It was determined that the region-based image identification methods are highly suitable for flood region detection. The first step in image processing was to convert the colour space from the RGB domain into a hue-saturation-value (HSV) domain. The $\mathrm{HSV}$ domain is preferred because it is more perceptually relevant than the Cartesian coordinates represented by the RGB domain. To distinguish a river object from its background, histogram equalisation was used to enhance the object contrast. The MS algorithm was then used to divide the video images into buildings, plants, rivers, and embankments. Finally, the RegGro algorithm was used to segment the images to form a binary mask. The outlines of the river areas were preserved within the binary mask. Figures 7 and 8 show example images from the two datasets obtained using the region-based image identification methods.

The flood alarm system captures surveillance images from remote monitoring stations, transmitting the flood monitoring information to an image server, and backing up the image data in an image database. The IFAM continually calculates the image data and automatically executes image dehazing procedures; it then executes the image analysis techniques to determine the water level of the current overflow range. Finally, the automatic floodalert monitoring system presents the detected results concerning the flooded areas. Hence, the proposed

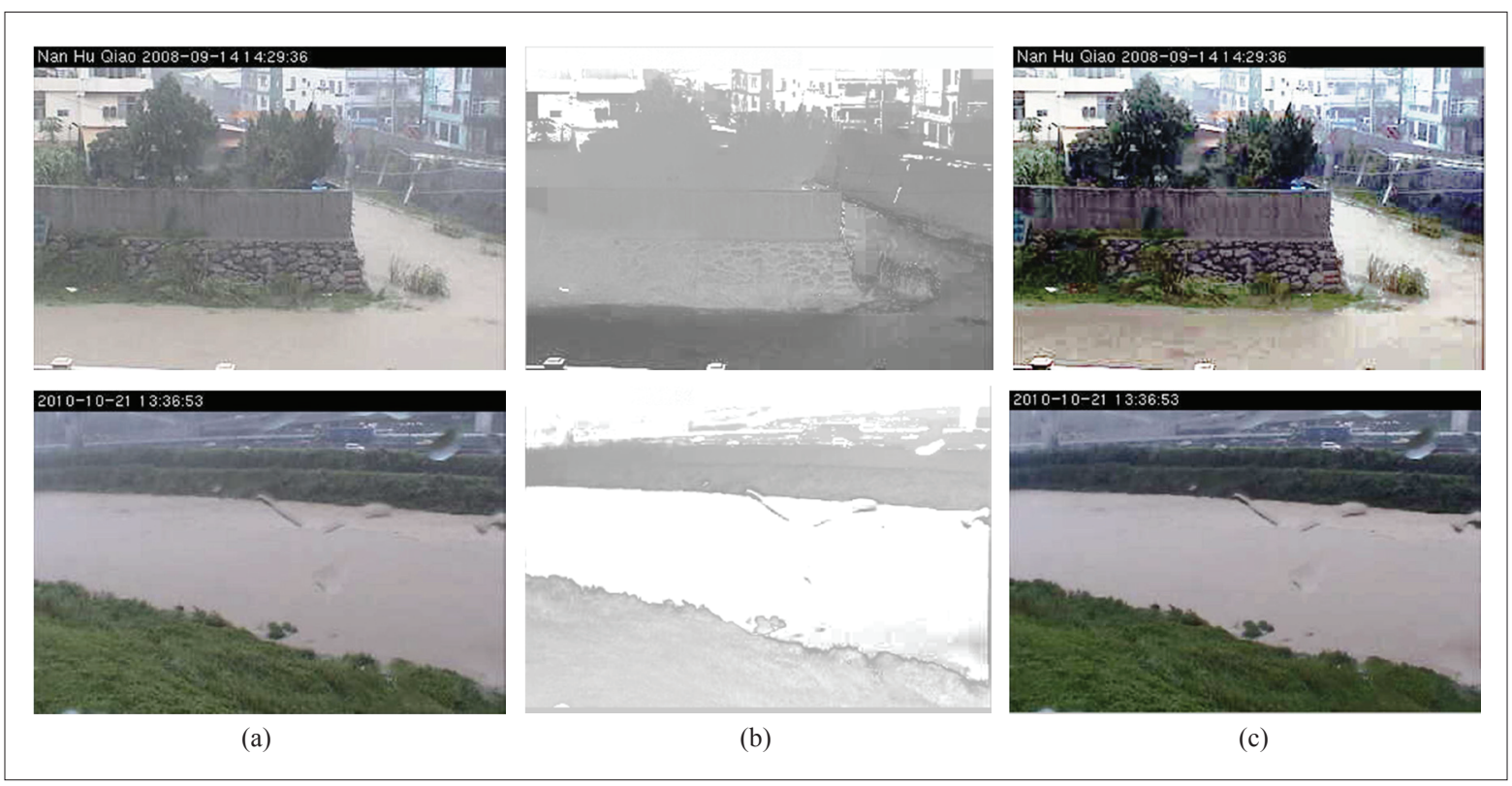

Figure 6: Image dehazing results: (a) original hazy images; (b) estimated transmission map and (c) restored haze-free images 

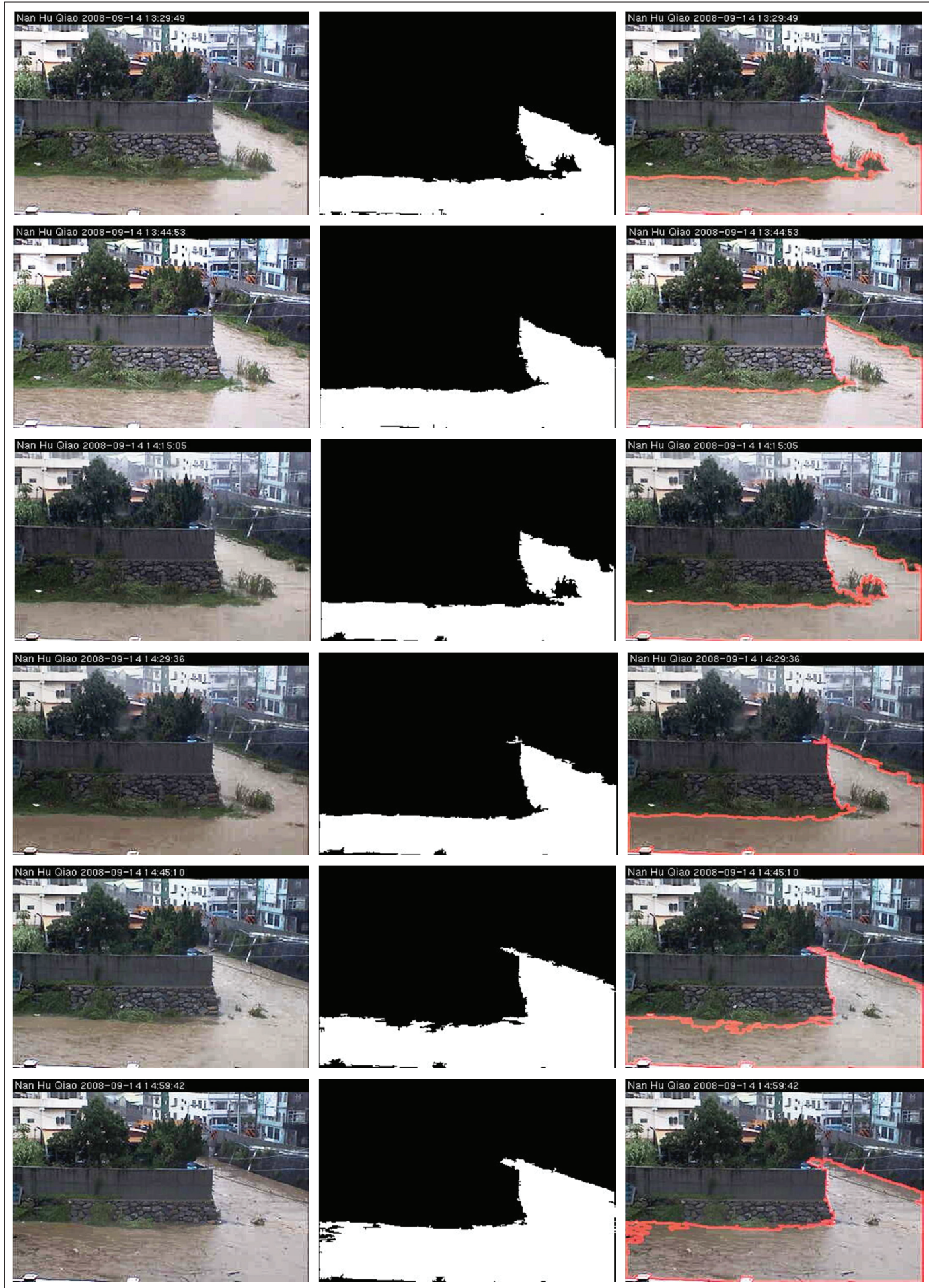

(a)

(b)

(c)

Figure 7: Surveillance image sequences of the Nan-Hu No. 2 Bridge. From left to right: (a) input haze-free images, (b) segmented images and (c) identified images 


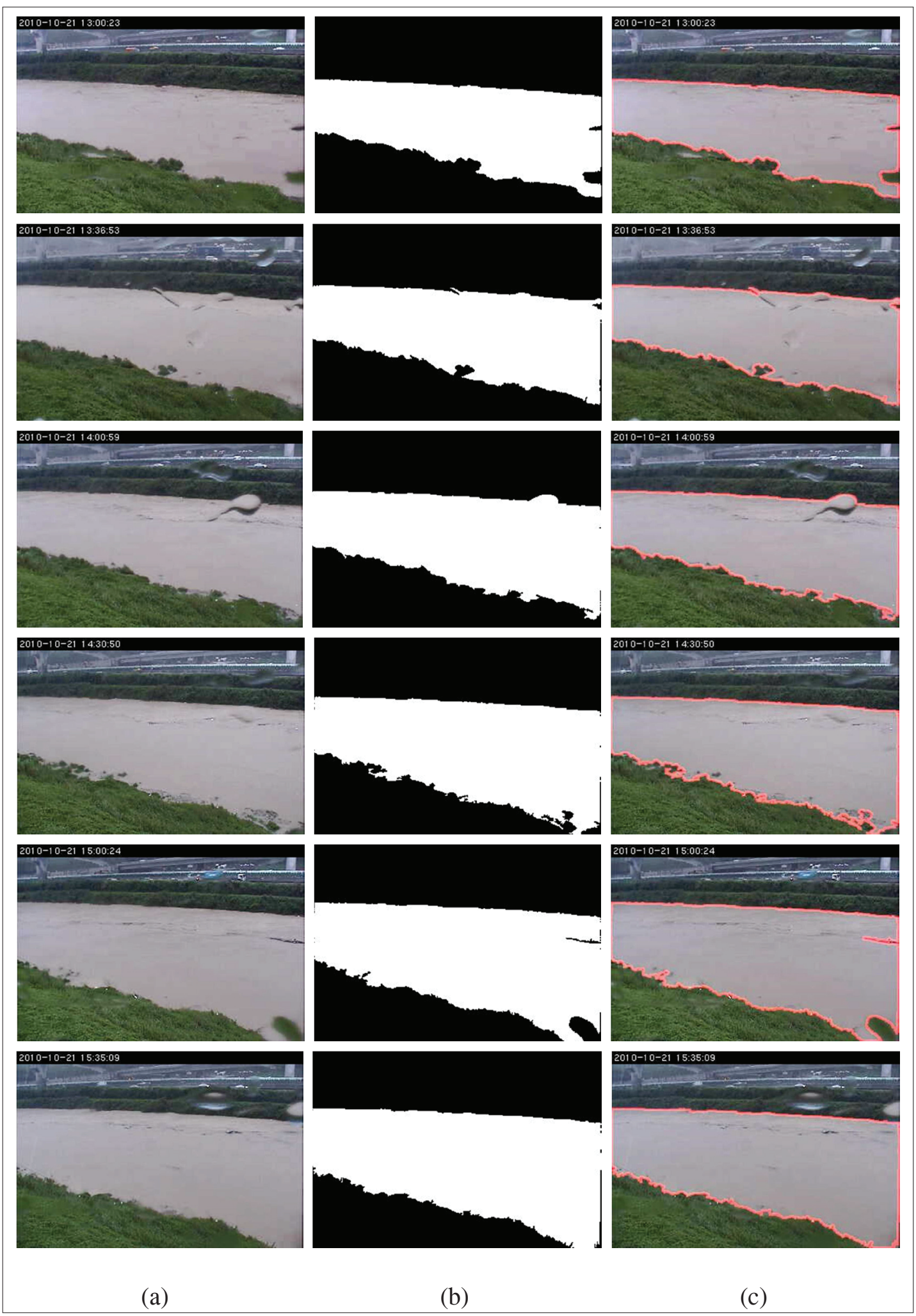

Figure 8: Surveillance image sequences of the Ma-Ling-Keng River. From left to right: (a) input haze-free images, (b) segmented images and (c) identified images 
system can provide real-time water resource information from all monitoring stations to multiple users, thereby supporting decision-related operations.

\section{CONCLUSION}

Image dehazing is a vital problem in monitoring systems. This paper proposes an automated single image dehazing method based on the dark channel prior, in which haze is considered the transmission. An IFAM is proposed in which an automated single image dehazing method and an image analysis technology are integrated for flood monitoring. The module is designed to improve the identification accuracy under adverse weather conditions while preserving the accuracy under clear weather conditions. Haze and rain, which strongly affect morphological features, are crucial features that should be evaluated for achieving high identification performance. The video surveillance images can be used for implementing an unattended monitoring system for detection and warning services. The experimental results demonstrate the suitability of the proposed automated IFAM, which can effectively detect and remove haze artefacts from a single image and identify flood hazard areas from image sequences.

\section{REFERENCES}

Abeyratne V.D.K. \& Ileperuma O.A. (2006). Air pollution monitoring in the city of Kandy: possible transboundary effects. Journal of the National Science Foundation of Sri Lanka 34(3): 137 - 141.

DOI: https://doi.org/10.4038/jnsfsr.v34i3.3644

Albanese A., Disabato F., Vigna R. \& Terzo O. (2008). Early warning system for flood events and automatic classification of low resolution satellite imagery: ITHACA approach. Proceedings of the International Workshop on Remote Sensing for Disaster Applications, Pavia, Italy, pp. $1-6$.

Attanayaka A. \& Wijeyaratne S.C. (2013). Corticolous lichen diversity, a potential indicator for monitoring air pollution in tropics. Journal of the National Science Foundation of Sri Lanka 41(2): 131 - 140.

DOI: https://doi.org/10.4038/jnsfsr.v41i2.5707

Barnum P.C., Narasimhan S. \& Kanade T. (2010). Analysis of rain and snow in frequency space. International Journal of Computer Vision 86(2 - 3): 256 - 274.

Basha E.A., Ravela S. \& Rus D. (2008). Model-based monitoring for early warning flood detection, Proceedings of the $6^{\text {th }}$ ACM Conference on Embedded Network Sensor Systems, 5 - 7 November, Raleigh, USA, pp. 295 - 308. DOI: https://doi.org/10.1145/1460412.1460442

Chang N. \& Guo D.H. (2006). Urban flash flood monitoring, mapping and forecasting via a tailored sensor network system. Proceedings of the 2006 IEEE International
Conference on Networking, Sensing and Control, 23 - 25 April, Ft. Lauderdale, FL, USA, pp. 757 - 761.

DOI: https://doi.org/10.1109/ICNSC.2006.1673241

Chen S.P. (1990). Remote Sensing Analysis in Geoscience. Beijing Mapping Press, Beijing, China.

Chen Z., Shen J. \& Roth P. (2013). Single image defogging algorithm based on dark channel priority. Journal of Multimedia 8(4): 432 - 438.

Fattal R. (2008). Single image dehazing. ACM Transactions on Graphics 27(3): 1 - 9 .

DOI: https://doi.org/10.1145/1360612.1360671

Gibson K.B., Võ D.T. \& Nguyen T.Q. (2012). An investigation of dehazing effects on image and video coding. IEEE Transactions on Image Processing 21(2): 662 - 673.

DOI: https://doi.org/10.1109/TIP.2011.2166968

Guo F., Tang J. \& Gai Z.X. (2014). Image dehazing based on haziness analysis. International Journal of Automation and Computing 11(1): $78-86$.

DOI: https://doi.org/10.1007/s11633-014-0768-7

He K., Sun J. \& Tang X. (2010). Single image haze removal using dark channel prior. IEEE Transactions on Pattern Analysis and Machine Intelligence 33(12): 2341 - 2353.

Hughes D., Greenwood P., Blair G., Coulson G., Pappenberger F., Smith P. \& Beven K. (2006). An intelligent and adaptable grid-based flood monitoring and warning system. Proceedings of the UK eScience All Hands Meeting, Nottingham, UK, pp. $53-60$.

Kang L.W., Lin C.W. \& Fu Y.H.(2012). Automatic single-imagebased rain streaks removal via image decomposition. IEEE Transactions on Image Processing 21(4): 1742 - 1755. DOI: https://doi.org/10.1109/TIP.2011.2179057

Krzhizhanovskaya V.V. et al. (15 authors) (2011). Flood early warning system: design, implementation and computational modules. Procedia Computer Science 4: 106 - 115. DOI: https://doi.org/10.1016/j.procs.2011.04.012

Krzysztofowicz R. \& Davis D.R. (1983). A methodology for evaluation of flood forecast-response systems: 2. Theory. Water Resources Research 19(6): 1431 - 1440. DOI: https://doi.org/10.1029/WR019i006p01431

Levin A., Lischinski D. \& Weiss Y. (2008). A closed-form solution to natural image matting. IEEE Transactions on Pattern Analysis and Machine Intelligence 30(2): $228-242$.

DOI: https://doi.org/10.1109/TPAMI.2007.1177

McCartney E.J. (1976). Optics of the Atmosphere: Scattering by Molecules and Particles. John Wiley and Sons, New York, USA.

Namer E., Shwartz S. \& Schechner Y.Y. (2009). Skyless polarimetric calibration and visibility enhancement. Optics Express 17(2): $472-493$.

DOI: https://doi.org/10.1364/OE.17.000472

Narasimhan S.G. \& Nayar S.K. (2003). Contrast restoration of weather degraded images. IEEE Transactions on Pattern Analysis and Machine Intelligence 25(6): 713 - 724.

DOI: https://doi.org/10.1109/TPAMI.2003.1201821

Patel S.P. \& Nakrani M. (2016). A review on methods of image dehazing. International Journal of Computer Applications 133(12): 975 - 8887.

Rani M., Kumar P., Yadav M. \& Hooda R.S. (2011). Wetland 
assessment and monitoring using image processing techniques: a case study of Ranchi, India. Journal of Geographic Information System 3(4): 345 - 350. DOI: https://doi.org/10.4236/jgis.2011.34032

Sapphaisal C. (2007). Forecasting and warning flood. Civil Engineering Magazine, volume 2, pp. 38 - 49. Engineering Institute of Thailand, Thailand.

Schechner Y.Y., Narasimhan S.G. \& Nayar S.K. (2003). Polarization-based vision through haze. Applied Optics 42(3): $511-525$.

DOI: https://doi.org/10.1364/AO.42.000511

Selvi C. \& Sathya S. (2014). Flood identification using satellite images. International Journal of Advanced Research in Electrical, Electronics and Instrumentation Engineering 3(1): $6497-6504$.

Shukla S. \& Pandey G.N. (2014). To design an architectural model for flood monitoring using wireless sensor network system. International Journal of Computer Science and Information Technologies 5(1): $502-507$.

Shwartz S., Namer E. \& Schechner Y.Y. (2006). Blind haze separation. Proceedings of the IEEE Conference on Computer Vision and Pattern Recognition, 17 - 22 July, New York, USA, pp. 1984 - 1991.

DOI: https://doi.org/10.1109/CVPR.2006.71

Tan Z., Bai X., Wang B. \& Higashi A. (2014). Fast single- image defogging. Fujitsu Scientific and Technical Journal 50(1): $60-65$.

Wang Y.F. (2009). Enhanced actions for water disaster warning system and evacuation. Proceedings of the Southeast Asia International Program (SEAIP), 30 November - 04 December, Taichung, Taiwan, pp.1 32 .

Wu J.H., Tseng C.H., Chen L.C., Lo S.W. \& Lin F.P. (2015). Automated image identification method for flood disaster monitoring in riverine environments: a case study in Taiwan. Proceedings of the AASRI International Conference on Industrial Electronics and Applications (IEA2015), London, UK, pp. 268 - 271.

Xiao C. \& Gan J. (2012). Fast image dehazing using guided joint bilateral filter. Visual Computer 28(6-8): 713 - 721 .

Zhai Y. \& Ji D. (2015). Single image dehazing for visibility improvement. Proceedings of the International Archives of the Photogrammetry, Remote Sensing and Spatial Information Sciences, Toronto, Canada, pp. 355 - 360.

Zhang J., Li L., Yang G., Zhang Y. \& Sun J. (2010). Local albedo-insensitive single image dehazing. Visual Computer 26(6-8): $761-768$.

Zhang J., Zhou C., Xu K. \& Watanabe M. (2002). Flood disaster monitoring and evaluation in China. Environmental Hazards 4(2): 33 - 43.

DOI: https://doi.org/10.3763/ehaz.2002.0404 